\title{
Optimisation of the transmit beam parameters for generation of subharmonic signals in native and altered populations of a commercial microbubble contrast agent SonoVue ${ }^{\circledR}$
}

\author{
Aoife M. Ivory \\ Trinity College Dublin, Ireland, aoife.ivory@npl.co.uk \\ James F. Meaney \\ Trinity College Dublin, Ireland \\ Andrew J. Fagan \\ Mayo Clinic, Rochester, MN, USA
}

See next page for additional authors

Follow this and additional works at: https://arrow.tudublin.ie/cieoart

Part of the Medicine and Health Sciences Commons

\section{Recommended Citation}

Aoife M. Ivory, James F. Meaney, Andrew J. Fagan, Jacinta E. Browne, Optimisation of the transmit beam parameters for generation of subharmonic signals in native and altered populations of a commercial microbubble contrast agent SonoVue ${ }^{\circledR}$, Physica Medica, Volume 70, 2020, Pages 176-183, ISSN 1120-1797, DOI: 10.1016/j.ejmp.2020.01.017.

This Article is brought to you for free and open access by the Centre for Industrial and Engineering Optics at ARROW@TU Dublin. It has been accepted for inclusion in Articles by an authorized administrator of ARROW@TU

Dublin. For more information, please contact

arrow.admin@tudublin.ie, aisling.coyne@tudublin.ie, gerard.connolly@tudublin.ie.

Funder: Irish Research Council

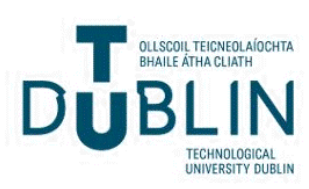


Authors

Aoife M. Ivory, James F. Meaney, Andrew J. Fagan, and Jacinta Browne

This article is available at ARROW@TU Dublin: https://arrow.tudublin.ie/cieoart/87 
Original paper

\title{
Optimisation of the transmit beam parameters for generation of subharmonic signals in native and altered populations of a commercial microbubble contrast agent SonoVue ${ }^{\circledR}$
}

\author{
Aoife M. Ivory ${ }^{\mathrm{a}, *}$, James F. Meaney ${ }^{\mathrm{a}}$, Andrew J. Fagan ${ }^{\mathrm{b}, 1}$, Jacinta E. Browne ${ }^{\mathrm{b}, \mathrm{c}, 1}$

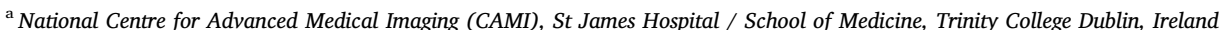 \\ ${ }^{\mathrm{b}}$ Department of Radiology, Mayo Clinic, Rochester, MN, USA \\ ${ }^{\mathrm{c}}$ School of Physics and Clinical \& Optometric Sciences, Medical Ultrasound Physics and Technology Group, Centre of Industrial Engineering Optics, FOCAS, Technical \\ University Dublin, Ireland
}

\section{A R T I C L E I N F O}

\section{Keywords:}

Ultrasound contrast agent

Acoustic characterisation

Subharmonic imaging

\begin{abstract}
A B S T R A C T
The aim of this work was to establish the optimum acoustic characterisation approach and insonation transmit beam parameters for subharmonic signal generation with 'native' and 'altered' populations of a commonly-used microbubble contrast agent. Dynamic contrast-enhanced (DCE) ultrasound is a non-invasive method of imaging the microvasculature, typically implemented using harmonic imaging. Subharmonic imaging, in which echoes at half the fundamental frequency are detected, detects signals which are generated by the ultrasound contrast agents (UCAs) but not by tissue. However, optimal transmission parameters and furthermore, the optimum acoustic characterisation method have not been established. The subharmonic response of 'native' and 'altered' UCA, altered through decantation, was investigated at transmit centre frequencies $1.8-5 \mathrm{MHz}$ and pulse lengths 1-8 cycles. The 'altered' UCA had reduced polydispersity (1-4 $\mu \mathrm{m}: 82 \%$ bubble volume), compared to 'native' (4-10 $\mu \mathrm{m}$ : 57\% bubble volume). A custom-built narrow-band acoustic characterisation system was found to be more appropriate for acoustic characterisation compared to the commonly used broadband pulse-echo approach. Both UCA generated the highest subharmonic signal at pulse length of 3-cycles. The maximum 'native' subharmonic signal was generated at a transmit centre frequency of $1.9 \mathrm{MHz}$, corresponding to a subharmonic at $0.95 \mathrm{MHz}$. This optimal frequency increased in the 'altered' population to $2.3-2.5 \mathrm{MHz}$, bringing the subharmonic above $1 \mathrm{MHz}$ and hence into a range amenable to clinical abdominal imaging transducers. The use of subharmonic signal detection coupled with a modified UCA size distribution has potential to significantly improve the quantification sensitivity and accuracy of DCE ultrasound imaging.
\end{abstract}

\section{Introduction}

Ultrasound contrast agents (UCA) used in contrast-enhanced ultrasound imaging improve the contrast-to-tissue ratio, particularly for difficult to image patients such as obese patients, via an increased difference in acoustic impedance between tissue and the blood with UCA compared to blood alone. SonoVue ${ }^{\circledR}$ (Bracco, Italy) is the only UCA currently licensed in Europe. UCA enhance the amplitude of the signal, resulting in an improved sensitivity to both deeper tissues and small vessels which might otherwise be beyond the detection capabilities of conventional ultrasound techniques. UCAs are micron-sized bubbles, typically comprised of a perfluorocarbon gas encapsulated in a phospholipid shell, and are thus highly compressible, producing nonlinear oscillations above certain acoustic pressure thresholds. This nonlinear behaviour leads to the generation of both harmonic and subharmonic signal reflections from the UCA. Harmonic imaging has been extensively researched and is implemented clinically for contrastenhanced ultrasound (CEUS) imaging, with applications to evaluate cardiac function through assessing pulmonary transmit stretch [1], and for characterisation of breast [2] and liver lesions [3,4]. However, in vivo the sensitivity of CEUS harmonic imaging is limited by the fact that there is also harmonic signal generation in tissue at the acoustic pressure levels used clinically in ultrasound imaging.

Subharmonic imaging (SHI), in which echoes at half the fundamental frequency are detected, has been suggested as an alternative to harmonic imaging for producing improved blood-to-tissue contrast, on

\footnotetext{
* Corresponding author.

E-mail address: aoife.ivory@npl.co.uk (A.M. Ivory).

${ }^{1}$ Joint senior authors.
} 

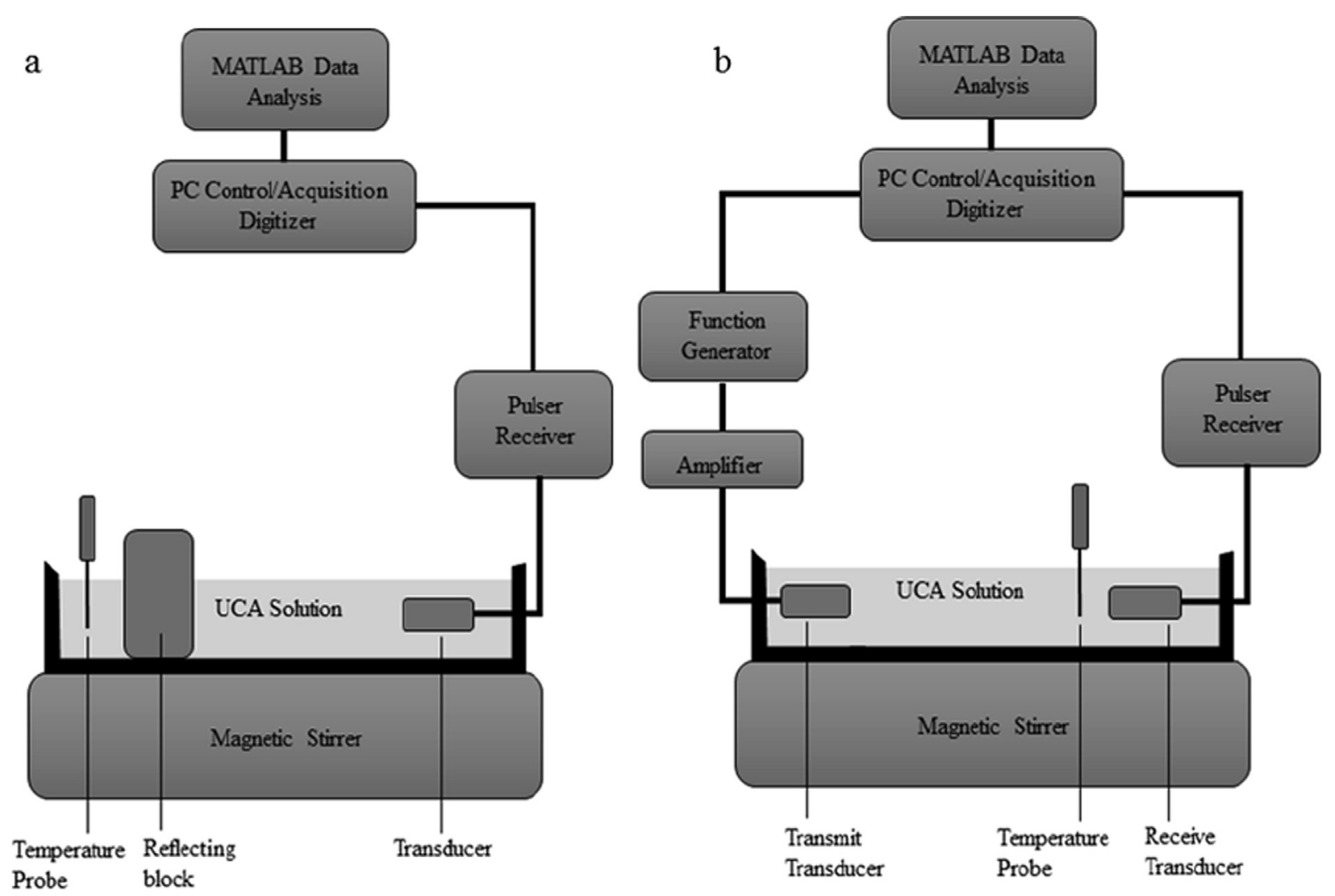

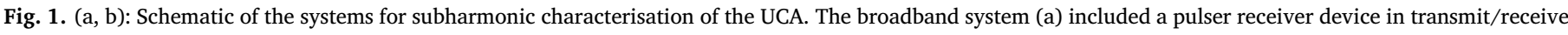

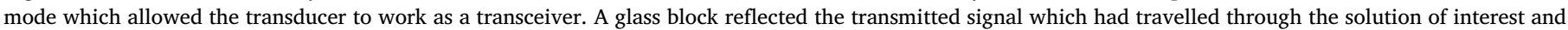

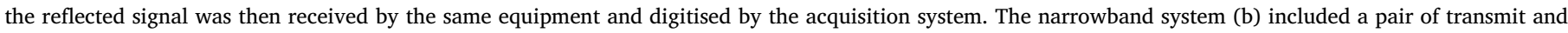

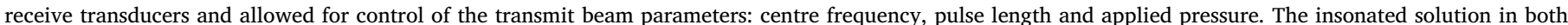
systems was constantly stirred to ensure that microbubbles did not float to the top or settle.

the basis that tissue does not produce significant subharmonic content [5-7]. Additionally, due to the frequency-dependent attenuation of ultrasound beam energy in tissue, the lower frequency subharmonic signals result in less attenuation of the signal. Early studies investigating the SHI approach have demonstrated that a significant amount of subharmonic signal can be generated at pressures typically used in conventional imaging, and with lower tissue attenuation of the signal, resulting in increased penetration depth $[5,6,8]$. The subharmonic response of UCAs depends on many factors, including controllable transmit beam parameters such as: the transmit centre frequency, the transmit pulse-length, and the applied acoustic pressure $[6,8-10]$. Investigation of the SHI response of a particular UCA requires full characterisation of its acoustic properties. However, there is no standardised methodology for acoustic characterisation, with broadband [11] and narrowband [12] approaches, relating to the pulse length of the excitation signal, described in the literature. A further confound is whether these approaches are performed in pulse-echo mode $[13,14]$, where the transducer acts as both the transmitter and receiver, or through-transmission mode [15], where both a transmit and receive transducer are positioned co-axially.

SHI is not currently implemented in the clinical environment; a necessary first step for its clinical implementation is to investigate optimal conditions for maximising the subharmonic signal from the UCA, for example to determine the optimum transmit parameters yielding maximum signal generation. Furthermore, commercial UCA are optimised for the current clinical applications using harmonic imaging approaches, with no prior attempt at optimisation for SHI. The aims of this study were threefold: to investigate the optimum acoustic characterisation method for the commercially available UCA used in the study, to fully characterise the subharmonic signal emitted by this UCA with a view to optimising the transmit beam properties to generate the maximum SH signal, and to investigate the effect of the UCA's microbubble size distribution on the SH signal.

\section{Methods}

To investigate the optimal conditions for subharmonic signal generation, experiments focused on two aspects:

(i) UCA microbubble size distribution - the size distribution of a commonly-available commercial UCA (SonoVue ${ }^{\circledR}$, Bracco, Italy) was modified, with experiments performed on 'native' and 'altered' distributions to investigate this effect on the frequency of maximum subharmonic signal generation.

(ii) Transmit beam properties - the transmit centre frequency and transmitted pulse length were varied to maximise the subharmonic signal magnitude. In all cases, the acoustic pressure level was maintained above the threshold for subharmonic signal generation but below that at which appreciable microbubble destruction is known to occur.

\subsection{Preparation of the UCA}

The preparation of the 'native' UCA solution was performed as per the manufacturer's instructions. The UCA was left to stand for ten minutes after reconstitution to allow the bubbles to stabilise, before samples were extracted from the main solution for subsequent characterisation and measurement.

The decantation method [13] was used to alter the UCA's size distribution and polydispersity. The size distribution of the 'native' and 
'altered' UCA populations were measured by electro-impedance volumetric zone sensing using a Coulter Counter ${ }^{\mathrm{TM}}$ (Beckman Coulter ${ }^{\mathrm{TM}}$, Life Sciences, USA), with a measurement range of $1.3-10 \mu \mathrm{m}$. The measurements were made for 3 vials with 5 sample measurements taken per vial. The total concentration, the size distribution and the volume distribution (based on the assumption of a spherical bubble) were then determined.

\subsection{Design and comparison of characterisation techniques}

A secondary aim of this study was to establish which type of system is more applicable for the characterisation of UCA. Broadband and narrowband transmit/receive bubble characterisation systems for investigating the bubble response to various transmit beam profiles were designed to perform a comparison of these techniques (Fig. 1). For the former, a broadband 'substitution method' pulse-echo system was developed. A pulser-receiver device (PR5052, Olympus, USA) was used to provide a single excitation pulse to a selected single element transducer (Olympus, USA); the signal passed through the UCA solution, was reflected by the glass block reflector and received by the same transducer. The echo-signals received by the transducer were captured and recorded by the acquisition system, which comprised a data acquisition card and code written in the LabVIEW programming environment (PCI-5144/LabVIEW, National Instruments, USA). The diecast box containing the UCA solution was positioned on a magnetic stirrer to maintain a uniform UCA solution and avoid microbubble floatation.

The narrowband system comprised of interchangeable single element transducers for transmit and receive functions, with their respective centre frequencies matched as closely as possible to the required centre transmit (1.8-5 MHz) and receive (subharmonic; 0.9-2.25 MHz) frequencies (Table 1). Sinusoidal waveforms of variable pulse-length (1-8 cycles) were produced using a waveform generator (AFG3251, Tektronix, USA) and amplified by a radiofrequency amplifier (75A250, Amplifier Research, USA) such that the maximum output acoustic pressure did not exceed $150 \mathrm{kPa}$ (the level found to not adversely affect the microbubbles). After passing through the UCA sample solution, the measured signal from the receive transducer was amplified by a pulser-receiver device (PR5052, Olympus, USA) configured in the 'isolated receive' mode. Digitisation of the analogue-amplified signal was carried out using a $250 \mathrm{MSample/s}$ analogue-to-digital converter using a PC-based data acquisition card (PCI-5144, LabVIEW ${ }^{\mathrm{rm}}$, National Instruments, USA). A trigger signal from the waveform generator was used to synchronise the data acquisition. Three independent samples of each population were measured 30 times at each set of transmit parameters.

Prior to the UCA measurements, the output pressure, transmit bandwidth and transmit/receive sensitivity of the single element transducers were accurately characterised using a calibrated $0.5 \mathrm{~mm}$ needle hydrophone (Precision Acoustics, UK). The hydrophone was used to measure the output of the transducers following excitation using the various transmit parameters, and a voltage-to-pressure conversion [16] was carried out using:

Table 1

Details of the single-element transducers used for UCA acoustic characterisation measurements, with the corresponding transmit centre frequency ranges used for the transmit and receive functions.

\begin{tabular}{|c|c|c|}
\hline $\begin{array}{l}\text { Transmit centre Frequency } \\
\text { Range }[\mathrm{MHz}]\end{array}$ & $\begin{array}{l}\text { Transmit: } \\
\text { model, centre } \\
\text { frequency }[\mathrm{MHz}]\end{array}$ & $\begin{array}{l}\text { Receive: } \\
\text { model, centre frequency } \\
{[\mathrm{MHz}]}\end{array}$ \\
\hline $1.8-2.8$ & V306, 2.25 & V303, 1.0 \\
\hline $2.9-3.75$ & V382, 3.5 & V306, 2.25 \\
\hline $4.5-5$ & V309, 5.0 & V382, 3.5 \\
\hline
\end{tabular}

$P(t)=F^{-1}\left\{\frac{F(V(t))}{M(f)}\right\}$

where $P$ is the pressure, $V$ is the voltage recorded, $M(f)$ the hydrophone frequency response and $F$ and $F^{-1}$ are the Fourier and inverse Fourier transforms (respectively). The transmit bandwidths defined by the fullwidth at half-maximum and the full-width at tenth- maximum were extracted from the transmit frequency spectra calculated using the Welch Periodogram [17] from the measured hydrophone data. Additionally, the measured signals from each transducer were corrected for the transducers' individual receive sensitivity profiles, as measured using the hydrophone method [18], and the differences in inter-transducer sensitivity, due to manufacturing variability of these transducers, was also taken into account by normalising the measured data to the maximum amplitude of the most sensitive transducer.

The amplitude of the UCA-measured waveforms were subsequently corrected for the transducer sensitivity, and spectral analysis of the corrected waveforms was performed using in-house developed code in MATLAB $^{\mathrm{TM}}$ (The Mathworks, USA) for Welch Periodogram analysis.

Both the broadband pulse-echo and narrowband through-transmission acoustic characterisation systems employed the 'substitution method' to measure the UCA attenuation $(\alpha)$ using:

$\alpha=\frac{10}{d} \log _{10}\left(\frac{I_{\text {sample }}}{I_{\text {water }}}\right)$

Where $I_{\text {sample }}$ is the mean of the power spectrum of the signal through UCA solution, $I_{\text {water }}$ is the mean of the power spectrum of the signal through deionised water, and $d$ is the distance travelled by the pulse. The commonly used broadband 'pulse-echo' system was compared to the narrowband 'through-transmission' acoustic characterisation system by measuring the attenuation as a function of concentration. Narrow bandwidths were produced using 8 cycle pulse lengths.

\subsection{Determination of the resonance frequency}

The narrowband system was then used to investigate resonance frequency by determining where the peak attenuation as a function of frequency occurred for each UCA population. The UCA solutions were stirred for approximately $30 \mathrm{~s}$ to ensure a homogeneous solution prior to the measurements and continuously thereafter, to ensure the UCA did not float or settle.

\subsection{Determination of optimum subharmonic response as a function of transmit centre frequency and pulse length}

In order to gain an understanding of the relationship between the subharmonic response and the various transmit parameters the subharmonic characterisation experiments focused on the 'native' UCA over a wide range of transmit parameters. The parameters investigated were then tailored to those demonstrating a higher probability of generating stronger subharmonic signals. The investigation of the 'altered' UCA was then included, which was designed with a size distribution to produce maximum subharmonic signals in a clinically relevant frequency range. The impact of the 'altered' UCA size distribution on optimising the subharmonic signals within the clinically chosen frequency range was investigated.

The subharmonic response of the 'native' UCA as a function of transmit beam pulse train length, corresponding to the inverse of the transmit bandwidth, was investigated at frequencies of $2.25,2.8,3.4$, 3.75, 4.5 and 5.0 MHz. These frequencies cover the range of those used clinically for liver examinations, which was the clinical application focused on for this investigation. Excitation pulse train lengths ranging from 1 to 8 cycles were investigated, corresponding to 8 different transmit bandwidths for each frequency. Samples of $150 \mu$ of UCA in $300 \mathrm{ml}$ of deionised water were measured for approximately $30 \mathrm{~s}$ at a PRF (pulse repetition frequency) of $1 \mathrm{~Hz}$. The UCA samples were 
replaced after each measurement, to ensure that the microbubbles were not affected by the previous insonation.

The subharmonic signal amplitude was then extracted from the data for all transmit frequencies investigated through integrating across the bandwidths defined as: half the transmit frequency's (i) full-width halfmaximum (henceforth called " $\mathrm{SH}_{\mathrm{FWHM}}$ "), and (ii) and full-width tenth-

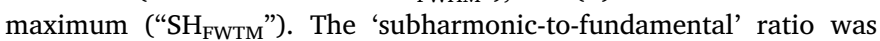
calculated from the frequency spectra for transmit centre frequencies of 3.4, 3.75, 4.5 and $5.0 \mathrm{MHz}$, for both the FWHM and FWTM bandwidths. Due to the sensitivity of the $1 \mathrm{MHz}$ receive transducer used when transmitting at 2.25 and $2.8 \mathrm{MHz}$, which did not capture the fundamental bandwidth, this was not possible for the lower frequencies.

To establish the frequency and bandwidth at which the 'native' population of the UCA produced the highest subharmonic signal, measurements were made with a transmit centre frequency of $1.8 \mathrm{MHz}$ to $3.7 \mathrm{MHz}$ in steps of $0.1 \mathrm{MHz}$ with pulse lengths of 3 and 8 cycles. Due to the decreased volume of UCA available, the subharmonic response of the 'altered' population as a function of frequency in the range of 1.9-3.9 MHz was investigated in steps of $0.2 \mathrm{MHz}$ at pulse lengths with 3 and 8 cycles. A $150 \mu$ sample was diluted in $300 \mathrm{ml}$ of deionised water for each measurement and, due to differences in the initial concentrations of the UCA, the subharmonic response of the 'native' and 'altered' distributions were measured at concentrations of $6.5 \times 10^{5} \mathrm{mb} \mathrm{ml}^{-1}$ and $1.2 \times 10^{5} \mathrm{mb} \mathrm{ml}^{-1}$ respectively.

Once the optimum frequency ranges for subharmonic generation were established, the subharmonic signal for the 'altered' population at these frequencies was measured at the concentration used for the characterisation of the 'native' population to allow for a direct comparison of the response of both populations.

\subsection{Determination of measurements uncertainty}

All measurements were repeated for three independent samples of UCA $(N=3)$, with approximately 30 measurements taken per sample solution $\left(n_{i}\right)$ for each transmit beam profile. A mean frequency spectrum was calculated for each sample and the mean value of interest was calculated $\left(\mu_{i}\right)$, e.g. for the subharmonic signal amplitude at $\mathrm{SH}_{\mathrm{FWHM}}$. The mean value $\left(\mu_{T}\right)$ of these measurements was then calculated across the three solutions (Eq. (3)) and presented with the standard error, $\widehat{\Delta \mu_{T}}$, between the measurements as the error on the measurements, (Eq. (4), where $s_{i}$ is the unbiased standard deviation of the sample):

$\widehat{\mu_{T}}=\frac{\sum_{i=1}^{N} n_{i} \mu_{i}}{\sum_{i=1}^{N} n_{i}}$

$\widehat{\Delta \mu_{T}}=\frac{\sqrt{\sum_{i=1}^{N} n_{i} s_{i}^{2}}}{\sum_{i=1}^{N} n_{i}}$

\section{Results}

\subsection{Preparation of the UCA}

Decantation of the 'native' population successfully resulted in an 'altered' population, as shown in Fig. 2. UCA distribution measurements showed the lower fraction of the vial contained mainly microbubbles of diameter $1-4 \mu \mathrm{m}$, accounting for $99 \%$ of the bubble count and $82 \%$ of the bubble volume. The increase in the number at the lower size range, in comparison to the 'native', is a result of the larger bubbles floating to the top of the available volume and pushing some of the smaller bubbles deeper into the vial. The average concentration of the 'altered' population was found to be $1.88 \times 10^{8} \mathrm{mb} \mathrm{ml}^{-1}$ (Table 2). The mean size based on number is similar between the two populations 1.85/ $1.8 \mu \mathrm{m}$ for the 'native' and 'altered' UCA respectively. The mean size based on the volume distribution indicated a larger difference changing from 4.0 to $2.5 \mu \mathrm{m}$ after decantation. The volume distribution has been linked to the efficacy of SonoVue ${ }^{\circledR}$ for fundamental imaging [19] although it remained to be established which estimation is more applicable for subharmonic efficacy. A variation in measured concentration was noted which is thought to be a result of vial-to-vial variability which has been noted in other studies [20].

\subsection{Comparison of the characterisation techniques}

The comparison of the narrowband versus broadband measurement of attenuation as a function of concentration is shown in Fig. 3. Both graphs show a linear increase in attenuation with concentration, in the range investigated. The broadband graph displays approximately 3-4 times higher values than the narrowband. The narrowband measurements are consistent with those previously published in the literature, e.g. $1.2 \mathrm{~dB} / \mathrm{cm}$ at $3 \mathrm{MHz}$ and $\sim 1.5 \times 10^{6} \mathrm{mb} \mathrm{ml}^{-1}$ [21]. Furthermore, broadband measurements resulted in higher variability in the attenuation measurements across all concentrations compared to the narrowband measurements as indicated by the standard error of the measurements represented by the error bars in the figure. The variability in the broadband measurements is thought to be a result of the relation between UCA response and insonation frequency. UCA are not monodisperse and the variability in the size distribution means that the resonance frequency is not the same for each individual microbubble, and therefore the response to any particular transmit frequency will not be the same for each individual microbubble. The broadband measurements included a wide range of frequencies, meaning that the response of the UCA was more variable than the narrowband measurements. The narrowband had less variation of transmit frequency and, therefore, resulted in a less variable response of the UCA, allowing a more accurate estimation of the attenuation of the UCA.

\subsection{Determination of the resonance frequency}

The attenuation as a function of frequency of 'native' and 'altered' UCA populations were measured using the narrowband system (Fig. 4). There are two main attenuation peaks between 1.8 and $2.4 \mathrm{MHz}$ and 3.1-3.6 $\mathrm{MHz}$ for the native population which correspond to the two peaks observed at $<2 \mu \mathrm{m}$ and $3-6 \mu \mathrm{m}$ in the volume distribution data. The peak in the attenuation spectrum indicates the resonance frequency $[14,22]$. The maximum attenuation measured as a function of frequency for the 'native' UCA population was found to be at $2.1 \mathrm{MHz}$. The maximum attenuation measured as a function of frequency for the 'altered' UCA population of $0.7 \pm 0.1 \mathrm{~dB} \mathrm{~cm}$ c $^{-1}$ was measured at $2.5 \mathrm{MHz}$. This supports the theory that, as the mean size decreases, the peak in attenuation coefficient occurs at a higher frequency, and that at less polydisperse size distributions, the peak in attenuation occurs in a narrower frequency range [23].

\subsection{Determination of optimum subharmonic response as a function of transmit centre frequency and pulse length}

The subharmonic response as a function of excitation pulse train length was then measured for the 'native' UCA. The measured subharmonic amplitude was integrated over the bandwidths $\mathrm{SH}_{\mathrm{FWHM}}$ $\left(\mathrm{SH}_{\mathrm{Amp} \text { FWHM }}\right)$ and for the wider frequency range at $\mathrm{SH}_{\mathrm{FWTM}}\left(\mathrm{SH}_{\mathrm{Amp}}\right.$ FWTM). There was overlap of the fundamental and subharmonic bandwidths for the FWHM and FWTM 1 cycle pulse length and at 2 cycle pulse length for the FWTM, and hence due to contamination of the subharmonic signals this data could not be compared. Across the six transmit centre frequencies, the response from the 3 cycle pulse length consistently resulted in the highest subharmonic signal level compared to the other pulse lengths investigated for both defined bandwidths. The $\mathrm{SH}_{\mathrm{FWTM}}$ amplitude was significantly higher than the $\mathrm{SH}_{\mathrm{FWHM}}$, which is to be expected considering the FWTM included more of the generated subharmonic signal. The subharmonic signal level increased 


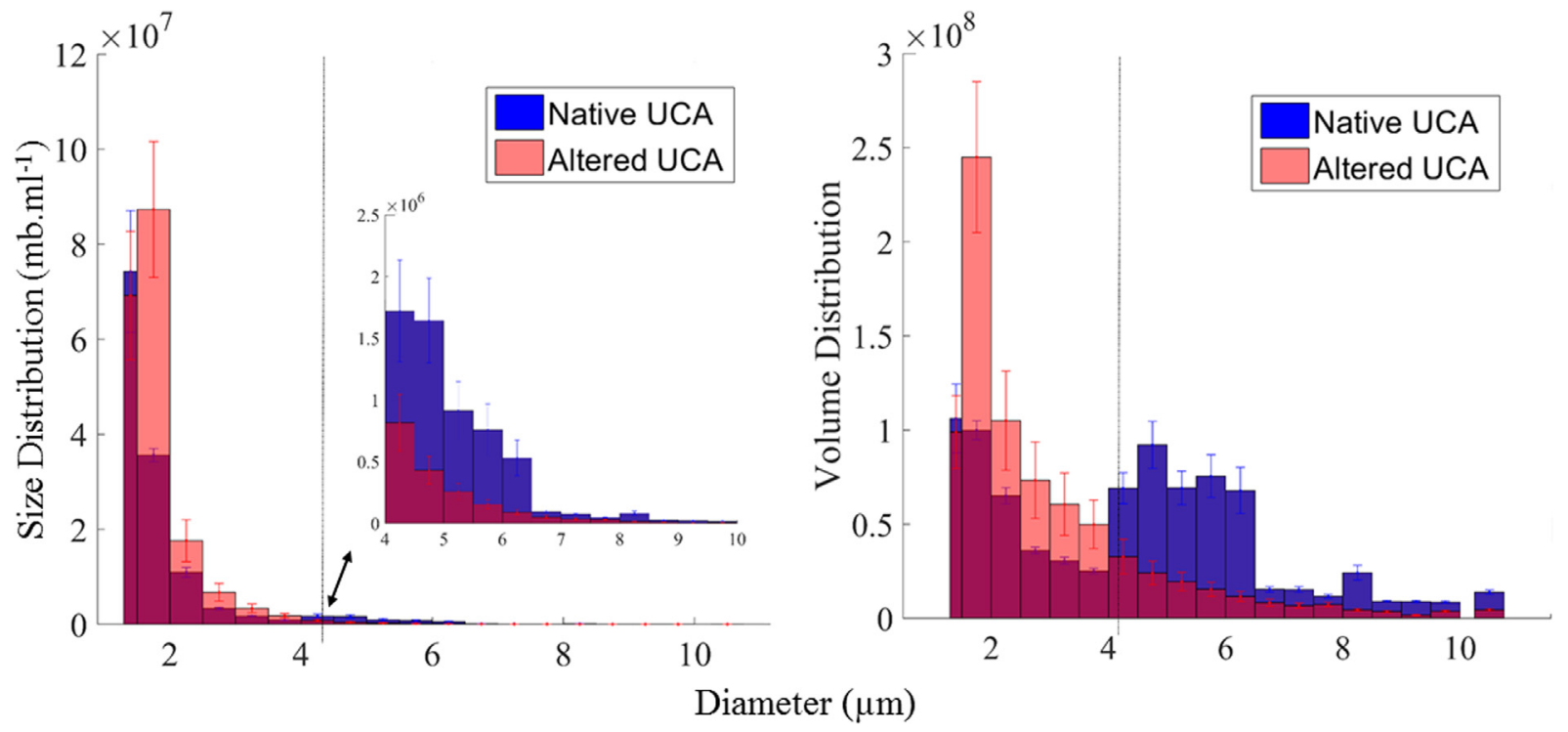

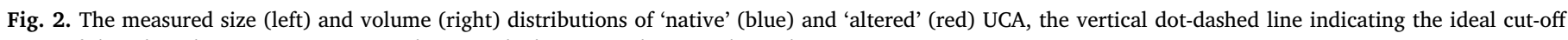
point of the 'altered' UCA (1-4 $\mu \mathrm{m})$ according to calculations made using the Stokes equation.

with decreasing transmit centre frequency, the highest signals were recorded at the lowest transmit frequency investigated $(2.25 \mathrm{MHz})$.

The subharmonic-to-fundamental ratio was also determined from the frequency spectra for the frequencies $3.4 \mathrm{MHz}, 3.75 \mathrm{MHz}, 4.5 \mathrm{MHz}$ and $5 \mathrm{MHz}$ over both the FWHM and FWTM bandwidths. There was a marginal increase in the subharmonic-to-fundamental ratio when the bandwidth is defined by the FWTM. This data also supported that the 3 cycles transmit pulse length appears to result in greater subharmonic signal generation for the 'native' UCA. The subharmonic-to-fundamental ratio (SH-F ratio) also increased with decreasing frequency.

The subharmonic signal amplitude across the bandwidth $\mathrm{SH}_{\mathrm{FWTM}}$, as a function of centre frequency and pulse length, corrected for the transducer receive sensitivity, is presented in Fig. 5; the amplitude across the $\mathrm{SH}_{\mathrm{FWHM}}$ bandwidth was also investigated but is not presented here. For the 'native' population the peak signal amplitude was measured at $1.9 \mathrm{MHz}$ for all pulse lengths and frequency bandwidths investigated. Similar to the 'native' measurements, the highest subharmonic amplitude for the 'altered' UCA was consistently found for a 3 cycle pulse length with the subharmonic bandwidth defined as $\mathrm{SH}_{\mathrm{FWTM}}$, with a peak subharmonic signal amplitude between 2.3 and $2.5 \mathrm{MHz}$ for $\mathrm{SH}_{\text {FWTM }}$. The narrower bandwidth, an 8 cycle pulse length, also had a peak in this frequency range near the resonance frequency given by the peak in the attenuation curve. Altering the population successfully narrowed the range of frequencies in which the subharmonic amplitude was highest, confining it to the higher end of the range measured in the native population (native: 1.9-2.5 MHz; and altered: $2.3-2.5 \mathrm{MHz}$ ). Both UCA indicated maximum subharmonic generation near the measured resonance frequency based on the attenuation spectra. Unlike the 'native' UCA, the entire optimised subharmonic frequency range for the 'altered' UCA lies within the clinically detectable frequency range used in abdominal imaging ( $>1 \mathrm{MHz}$ ).

It was predicted that the maximum subharmonic signal generated

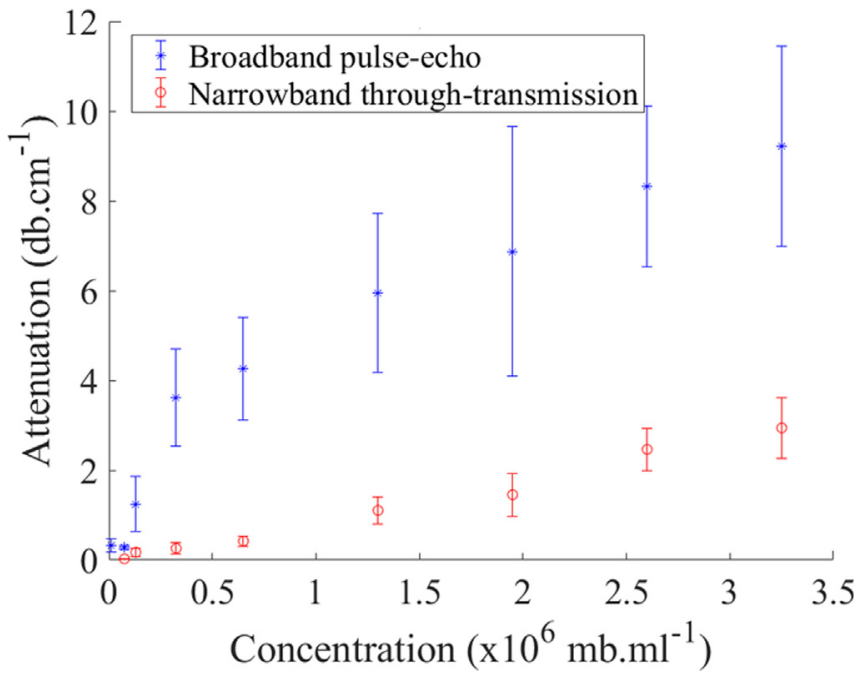

Fig. 3. Attenuation of the 'native' UCA measured as a function of concentration using a broadband and a narrowband characterisation system. Both systems show a linear increase in attenuation with concentration. The narrowband system shows less variability in the measurements (error bars) and resulted in attenuation values consistent with those published in literature.

using the 'altered' UCA would be greater than that from the 'native', due to more of the UCA sample being insonated at or near the optimum transmit centre frequency (i.e. the resonance frequency) in the less polydisperse 'altered' UCA. With the optimum transmit frequencies established both UCA were interrogated at the same concentration to compare the subharmonic signal generation. However, the absolute maximum subharmonic amplitude at the individual transmit centre

Table 2

The measured concentration and mean size of the 'native' and 'altered' SonoVue ${ }^{\circledR}$ UCA formulations.

\begin{tabular}{|c|c|c|c|c|c|c|}
\hline \multirow[t]{2}{*}{ Formulation } & \multicolumn{4}{|l|}{$\begin{array}{l}\text { Concentration } \\
{\left[\mathrm{mb} \mathrm{ml}^{-1}\right]}\end{array}$} & \multicolumn{2}{|l|}{ Mean Size $[\mu \mathrm{m}]$} \\
\hline & Vial 1 & Vial 2 & Vial 3 & Mean & based on number & based on volume \\
\hline 'native' UCA & $1.48 \pm 0.1 \times 10^{9}$ & $3.47 \pm 0.2 \times 10^{9}$ & $1.21 \pm 0.1 \times 10^{9}$ & $2.05 \pm 0.2 \times 10^{9}$ & 1.85 & 4.00 \\
\hline 'altered' UCA & $1.69 \pm 0.2 \times 10^{8}$ & $5.81 \pm 0.5 \times 10^{7}$ & $3.44 \pm 0.7 \times 10^{8}$ & $1.88 \pm 0.7 \times 10^{8}$ & 1.80 & 2.8 \\
\hline
\end{tabular}




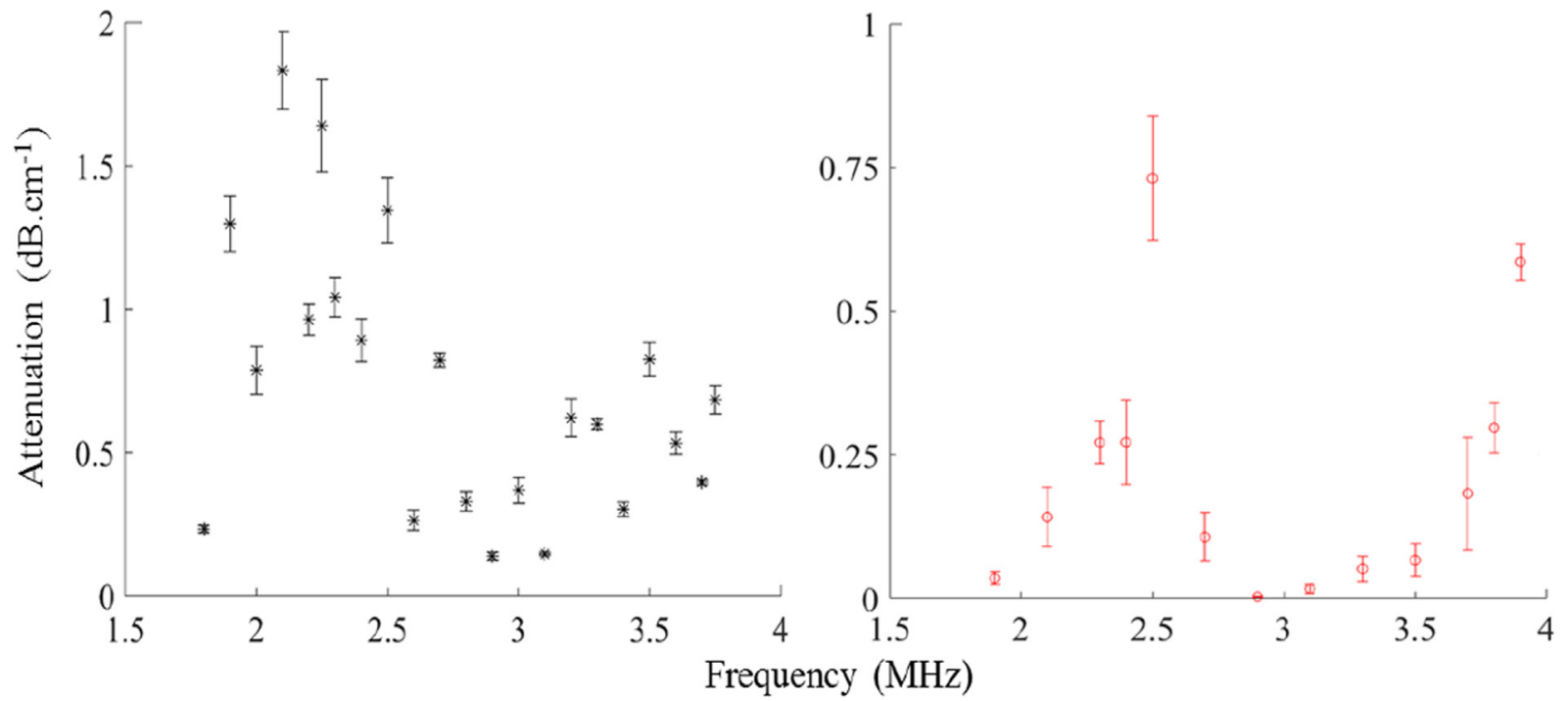

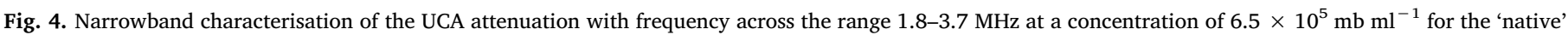

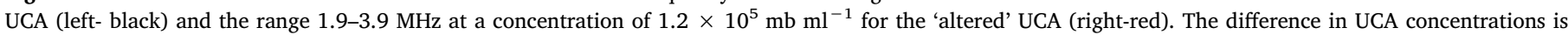
reflected in the different range of attenuation values measured in each population.

frequencies was higher in the 'native' (466 $\left.\pm 3 \mathrm{~V}^{2} / \mathrm{MHz}\right)$ than the 'altered' UCA ( $\left.400 \pm 3 \mathrm{~V}^{2} / \mathrm{MHz}\right)$, with the peaks occurring at $1.9 \mathrm{MHz}$ and $2.3 \mathrm{MHz}$ respectively. Fig. 6 shows the two regions of highest subharmonic generation and it can be seen that the 'altered' UCA resulted in similar subharmonic amplitudes to the 'native' UCA at 2.3-2.5 MHz.

\section{Discussion}

The generation of subharmonic signals from UCAs has been known for many years, although optimum transmit parameters have not yet been established for subharmonic abdominal imaging, nor indeed have UCA formulations been designed with subharmonic imaging in mind.
The current study aimed to address these limitations by characterising the subharmonic generation from 'native' and 'altered' populations generated by a commercially available clinical UCA, using a custombuilt narrowband characterisation system, operating over the frequency range 1-6 MHz typically used in abdominal imaging. The method of defining the generated subharmonic bandwidth was also optimised.

An 'altered' UCA, containing microbubbles in the size range of 1-4 $\mu \mathrm{m}$, was obtained through decantation [13], it was expected that this narrower size range would have two main effects on the subharmonic generation. Firstly, that the frequency at which the highest subharmonic signal was generated would be shifted up into the clinically-detectable range for abdominal imaging, namely, between 1 and $6 \mathrm{MHz}$. Secondly, that the more monodisperse size distribution of the
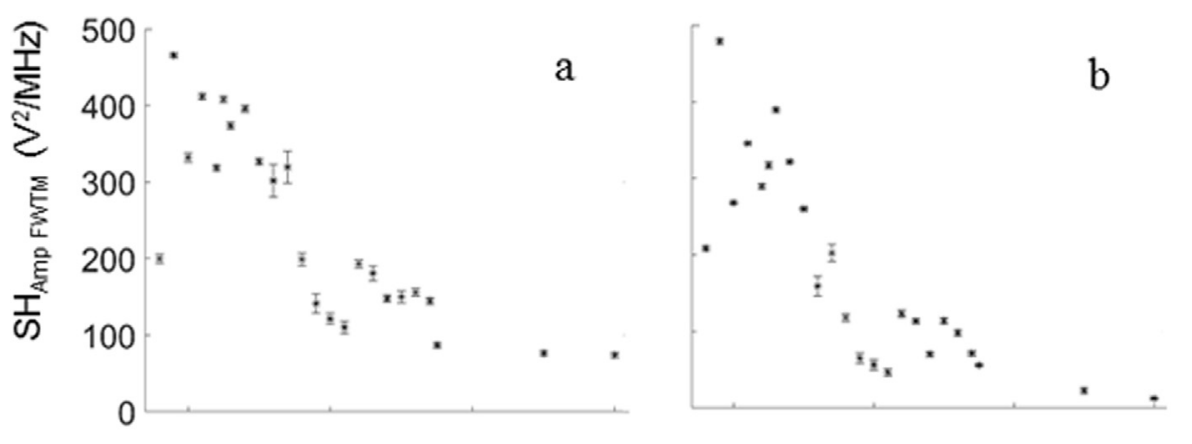

Fig. 5. Subharmonic signal amplitude at $\mathrm{SH}_{\mathrm{FWTM}}$ $\left(\mathrm{SH}_{\mathrm{Amp} \text { FWTM }}\right)$ for the 'native' UCA at concentration $6.5 \times 10^{5} \mathrm{mb} \mathrm{ml}^{-1}$ at pulse length 3 cycles (a) and 8 cycles (b) and the 'altered' UCA at concentration $1.2 \times 10^{5} \mathrm{mb} \mathrm{ml}^{-1}$ at pulse length 3 cycles (c) and 8 cycles (d) measured at an applied pressure $150 \mathrm{kPa}$. The data represents a mean of 3 solutions, insonated approximately 30 times at each transmit centre frequency. The data has been corrected for intra-transducer and inter-transducer sensitivity.

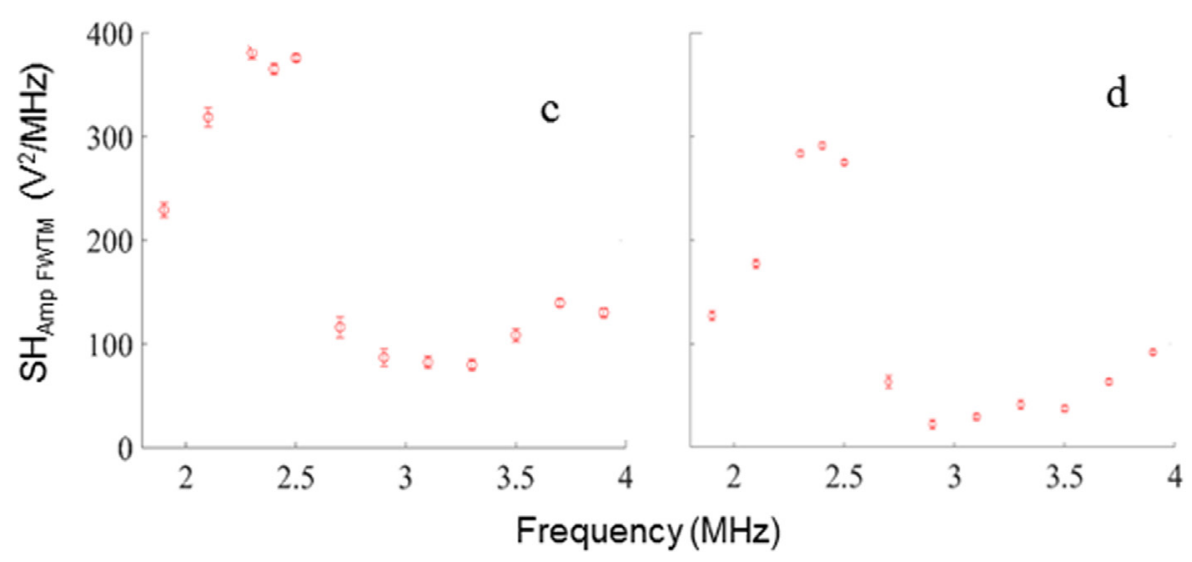




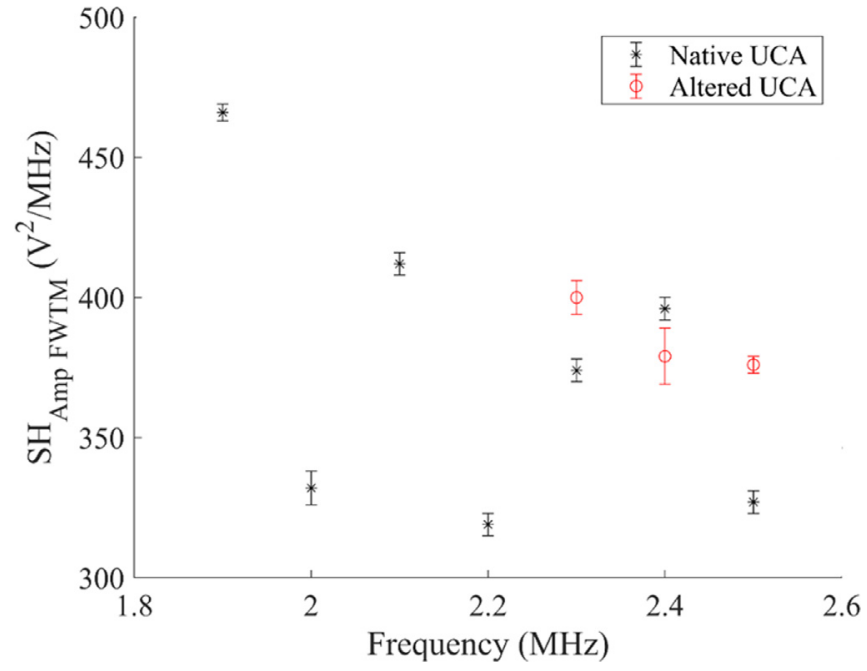

Fig. 6. Subharmonic amplitude at $\mathrm{SH}_{\mathrm{FWTM}}\left(\mathrm{SH}_{\mathrm{Amp}}\right.$ FWTM $)$ for the 'Native' and 'Altered' UCA at the transmit centre frequencies which gave the highest subharmonic signal, 1.9-2.5 $\mathrm{MHz}$ and 2.3-2.5 MHz, respectively. The data represents a mean of 3 solutions, insonated approximately 30 times at each transmit centre frequency.

UCAs would result in a higher subharmonic signal close to their resonance frequency, where the maximum subharmonic generation was expected, compared to the polydisperse 'native' UCA for the same applied pressure and concentration.

UCA microbubbles' exhibit an inherent resonance frequency which is a function of various physical and ambient parameters, such as: bubble radius; ambient pressure; liquid density; and viscoelastic stresses generated in encapsulation, effective surface tension and interfacial dilational surface viscosity [24]. The resonance frequency has been reported to affect the transmit centre frequency at which the maximum subharmonic signal is generated, where the subharmonic generation threshold is at a minimum. This was reported to be at twice the resonance frequency in both computational studies and experimentally $[25,26]$. However, more recently, in a numerical investigation Katiyar et al. [24] discussed a minimum threshold for subharmonic generation, which is not necessarily at the resonance frequency, but can occur in the range of the resonance frequency to twice the resonance frequency.

Although the resonance frequency is a fundamental parameter dictating the generation of efficient UCA response, there is disagreement in the literature of the resonance frequency for many commercial UCAs. The range of resonance frequencies for SonoVue ${ }^{\circledR}$ reported in the literature may be attributable to variations in measurement techniques. Using optical methods the resonance frequency of individual SonoVue ${ }^{\circledR}$ microbubbles with diameters 4, 3.2 and $2.6 \mu \mathrm{m}$ [27] have been reported as 1.6, 2.1 and $3.1 \mathrm{MHz}$, respectively. Other techniques include using backscatter measurements and attenuation measurements, the same group have reported a resonance frequency of $3 \mathrm{MHz}$ and $1.6 \mathrm{MHz}$ $[21,22]$ for these techniques respectively. Another study determined that the resonance frequency of SonoVue ${ }^{\circledR}$ was at $1.75 \mathrm{MHz}$ [28] using a narrowband through transmission system, although the authors did not state how they measured the resonance frequency.

It is also likely that measurement of the resonant frequency is strongly influenced by the polydispersity of commercial UCA; for example, Parrales et al. attributed such difficulties in identifying the resonance peak from attenuation measurements made using a broadband 'pulse-echo' system similar to that described herein to polydispersity [14]. SonoVue ${ }^{\circledR}$ is an extremely nonlinear agent which shows substantial harmonics/subharmonics at very low acoustic pressure ( $<50 \mathrm{kPa}$ ). This could particularly complicate broadband attenuation measurements because of the poor control of frequency-dependent transmit pressure [29].

In the current study, significant differences in attenuation measurements were measured using the broadband and narrowband systems, highlighting the importance of establishing a standardized measurement approach. The data presented herein support the preferential use of narrowband techniques for UCA characterization, in particular for the current commercial UCAs with a high degree of polydispersity. The narrowband attenuation measurements as a function of frequency indicated that the 'altered', more monodisperse population, UCA had a narrower attenuation spectrum. The peak of the attenuation spectrum indicates the resonance frequency $[14,23]$ which was found to increase from $2.1 \mathrm{MHz}$ for the 'native' UCA to $2.5 \mathrm{MHz}$ for the 'altered' UCA.

The subharmonic data measured using the narrowband approach did not include any overlap between the fundamental and subharmonic bandwidth which tends to be present in broadband transmit frequencies. In the literature, there is little discussion of the manner in which the subharmonic frequency bandwidth is determined, and rather the centre frequency is usually only reported. When the frequencies of interest, i.e. the fundamental and subharmonic bandwidths, are defined on the basis of the FWTM, in general there was a notable increase in the subharmonic signal amplitude and a slight rise in the subharmonic-tofundamental ratio. The increases noted when the FWTM definition was used, particularly the significant increase in the subharmonic amplitude, suggests that more of the generated subharmonic was included in this wider definition of the subharmonic frequencies.

It was found that SonoVue ${ }^{\circledR}$ responded with the highest subharmonic signal when insonated at 3 cycles, in agreement with Liu et al.'s study using a clinical scanner [10]. This may be due to a tradeoff between insonating the UCA with sufficient cycles to generate the subharmonic component and potential bubble shell alteration caused by insonating with longer pulse lengths. It was also noted that the subharmonic signal increased with decreasing centre frequency, supporting the theory that there is a lower subharmonic generation threshold near the measured resonance frequency rather than at twice the resonance frequency $[30,31]$. Similarly the subharmonic-to-fundamental ratio, another measure of subharmonic signals [32], was found to give the highest ratio at 3 cycles with increasing values as the centre frequency decreased.

Chomas et al. [7] defined the two subharmonic generation regimes: transmitting at resonance frequency (TR) and twice the resonance frequency (T2R). In the TR subharmonic regime, the microbubble is compressed below its resting radius every cycle of insonation but exhibits a maximum expansion on every other cycle, whereas in the T2R regime, the bubble is compressed below resting radius and exhibits maximum expansion every other cycle. Strong subharmonic generation was predicted theoretically and shown experimentally to be present in the T2R regime, when UCA were insonated at twice the resonance frequency [33]; more recently, it has been reported that there is a minimum threshold for subharmonic generation at twice the resonance frequency [34]. However, further studies have also shown lower subharmonic generation thresholds in the TR regime rather than in the T2R regime $[30,31]$, which also depends on the bubble size distribution [24]. Shekhar found that there was a vial-to-vial variability in contrast agent's size distributions lead to considerable differences in subharmonic imaging performance, and suggested that the subharmonic response could be considerably improved by modifying the size distribution [35]. In the current study, it was expected that altering the UCA to a narrower size distribution and insonating at the centre frequency with the highest subharmonic component would result in a higher signal level compared to the more disperse 'native' UCA, as more of the microbubbles would be insonated close to their resonance frequency. However, when comparing the signals generated using the optimum transmit parameters on each UCA, the subharmonic signal levels in the frequency range of $2.3-2.5 \mathrm{MHz}$ were found to be similar for both UCA populations. The subharmonic generation for both UCA populations was greatest when transmitting near the resonance 
frequency based on the attenuation measurements, supporting the theory of a lower subharmonic generation threshold at this frequency.

The requirement to tailor UCA such that their optimum subharmonic frequency ranges falls within that of clinical scanners was demonstrated in this study. The alteration of the SonoVue ${ }^{\circledast}$ formulation to a smaller bubble size and narrower size distribution succeeded in shifting the frequency at which the optimum subharmonic signal was generated to above $1 \mathrm{MHz}$, traditionally the lower limit for abdominal transducers. The narrower frequency range of the attenuation peak and the subharmonic peak may give rise to more reproducible and quantifiable signals in the future development of clinically applicable subharmonic imaging techniques, due to the reduction in the uncertainty in the Time Intensity Curve analysis and parameter measurement from a population of polydisperse UCA.

\section{Conclusions}

This study presents a comprehensive investigation of the characterisation and subharmonic response of both 'native' and 'altered' populations of a commonly-used UCA. A narrowband 'through-transmission' characterisation technique was found to be more appropriate for UCA characterisation than the commonly employed broadband 'pulse-echo' technique. The attenuation data presented herein indicated that a narrower attenuation peak is present for less polydisperse populations, which furthermore is at a maximum at the resonance frequency of the UCA. For both UCA populations, the largest subharmonic response was present when insonated with a transmitted 3 cycle pulse length near the resonance frequency of the particular contrast agent population. Reducing the mean size of the altered population, resulted in an increased resonance frequency and hence a shift in the frequency at which the maximum subharmonic occurred to the clinically detectable range for abdominal imaging. The established optimum transmit parameters and defined bandwidth for subharmonic signal detection can be readily translated to a clinical set-up, thereby allowing for a clinically applicable subharmonic imaging technique to be developed for the ultrasound contrast agent, SonoVue ${ }^{\circledR}$.

\section{Acknowledgements}

The authors would like to acknowledge funding from the Irish Research Council (GOIPG/2014/1323) and experimental support from Bracco Spa, Italy. Also, the authors would like to thank the Centre for Advanced Medical Imaging, St James's Hospital, Dublin and the FOCAS research institute of TU Dublin for access to core equipment facilities. In particular we would like to thank Dr. Alan Casey for his assistance with the use of the Coulter Counter ${ }^{\mathrm{TM}}$.

\section{References}

[1] de Lepper AGW, Herold IHF, Saporito S, Bouwman RA, Mischi M, Korsten HHM, et al. Noninvasive pulmonary transit time: a new parameter for general cardiac performance. Echocardiography 2017;34:1138-45.

[2] Chen M, Wang WP, Jia WR, Tang L, Wang Y, Zhan WW, et al. Three-dimensional contrast-enhanced sonography in the assessment of breast tumor angiogenesis: correlation with microvessel density and vascular endothelial growth factor expression. J Ultrasound Med 2014;33:835-46.

[3] Dietrich CF. Characterisation of focal liver lesions with contrast enhanced ultrasonography. Eur J Radiol 2004;51(Suppl):S9-17.

[4] Claudon M, Cosgrove D, Albrecht T, Bolondi L, Bosio M, Calliada F, et al. Guidelines and good clinical practice recommendations for contrast enhanced ultrasound (CEUS) - update 2008. Ultraschall 2008;29:28-44.

[5] Shankar PM, Dala Krishna P, Newhouse VL. Advantages of subharmonic over second harmonic backscatter for contrast-to-tissue echo enhancement. Ultrasound Med Biol 1998;24:395-9.

[6] Forsberg F, Shi WT, Goldberg BB. Subharmonic imaging of contrast agents
Ultrasonics 2000;38:93-8.

[7] Chomas J, Dayton P, May D, Ferrara K. Nondestructive subharmonic imaging. IEEE Trans Ultrason Ferroelectr Freq Control 2002;49:883-92.

[8] Harput S, Arif M, McLaughlan J, Cowell DM, Freear S. The effect of amplitude modulation on subharmonic imaging with chirp excitation. IEEE Trans Ultrason Ferroelectr Freq Control 2013;60:2532-44.

[9] Shi W, Forsberg F, Raichlen J, Needleman L, Goldberg B. Pressure dependence of subharmonic signals from contrast microbubbles. Ultrasound Med Biol 1999;25:275-83.

[10] Liu JB, Nio AQX, Esposito C, Jie C, Jie Z, Xing Z, et al. Characterizing the subharmonic response of four new microbubble formulations compared with three commercially-available ultrasound contrast agents. IEEE Int Ultrason Symp (IUS) 2016:1-4.

[11] Chatterjee D, Sarkar K, Jain P, Schreppler NE. On the suitability of broadband attenuation measurement for characterizing contrast microbubbles. Ultrasound Med Biol 2005;31:781-6.

[12] Chen Q, Zagzebski J, Wilson T, Stiles T. Pressure-dependent attenuation in ultrasound contrast agents. Ultrasound Med Biol 2002;28:1041-51.

[13] Goertz DE, de Jong N, van der Steen AF. Attenuation and size distribution measurements of definity and manipulated definity populations. Ultrasound Med Biol 2007;33:1376-88.

[14] Parrales MA, Fernandez JM, Perez-Saborid M, Kopechek JA, Porter TM. Acoustic characterization of monodisperse lipid-coated microbubbles: relationship between size and shell viscoelastic properties. J Acoust Soc Am 2014;136:1077.

[15] Faez T, Goertz D, De Jong N. Characterization of definity ultrasound contrast agent at frequency range of 5-15 MHz. Ultrasound Med Biol 2011;37:338-42.

[16] Hurrell A. Voltage to pressure conversion: are you getting 'phased' by the problem? J Phys Conf Ser 2004;1:57.

[17] Welch D, The P. Use of fast Fourier transform for the estimation of power spectra: a method based on time averaging over short, modified periodograms. IEEE Trans Audio Electroacoust 1967;15:70-3.

[18] Xiao D, Fan Q, Xu C, Zhang X. Measurement methods of ultrasonic transducer sensitivity. Ultrasonics 2016;68:150-4.

[19] Gorce JM, Arditi M, Schneider M. Influence of bubble size distribution on the echogenicity of ultrasound contrast agents: a study of SonoVue. Invest Radiol 2000;35:661-71.

[20] Sun C, Panagakou I, Sboros V, Butler MB, Kenwright D, Thomson AJ, et al. Influence of temperature, needle gauge and injection rate on the size distribution, concentration and acoustic responses of ultrasound contrast agents at high frequency. Ultrasonics 2016;70:84-91.

[21] de Jong N, Emmer M, van Wamel A, Versluis M. Ultrasonic characterization of ultrasound contrast agents. Med Biol Eng Comput 2009;47:861-73.

[22] Emmer M, Vos HJ, Goertz DE, van Wamel A, Versluis M, de Jong N. Pressuredependent attenuation and scattering of phospholipid-coated microbubbles at low acoustic pressures. Ultrasound Med Biol 2009;35:102-11.

[23] Gong Y, Cabodi M, Porter TM. Measurement of the attenuation coefficient for monodisperse populations of ultrasound contrast agents. IEEE Eng Med Biol Soc Annual Conf 2009:1964-6.

[24] Katiyar A, Sarkar K. Excitation threshold for subharmonic generation from contrast microbubbles. J Acoust Soc Am 2011;130:3137-47.

[25] Shankar PM, Krishna PD, Newhouse VL. Subharmonic backscattering from ultrasound contrast agents. J Acoust Soc Am 1999;106:2104-10.

[26] Kimmel E, Krasovitski B, Hoogi A, Razansky D, Adam D. Subharmonic response of encapsulated microbubbles: conditions for existence and amplification. Ultrasound Med Biol 2007;33:1767-76.

[27] Meer SMVD, Versluis M, Lohse D, Chin CT, Bouakaz A, Jong Nd. The resonance frequency of SonoVue as observed by high-speed optical imaging. IEEE Ultrason Symp 2004:1:343-5.

[28] Tang MX, Eckersley RJ. Nonlinear propagation of ultrasound through microbubble contrast agents and implications for imaging. IEEE Trans Ultrason Ferroelectr Freq Control 2006;53:2406-15.

[29] Frinking PJA. Communication with Bracco Suisse SA Personal Communication 2018.

[30] Faez T, Emmer M, Docter M, Sijl J, Versluis M, de Jong N. Characterizing the subharmonic response of phospholipid-coated microbubbles for carotid imaging. Ultrasound Med Biol 2011;37:958-70.

[31] Katiyar A, Sarkar K. Effects of encapsulation damping on the excitation threshold for subharmonic generation from contrast microbubbles. J Acoust Soc Am 2012;132:3576-85.

[32] Andersen KS, Jensen JA. Impact of acoustic pressure on ambient pressure estimation using ultrasound contrast agent. Ultrasonics 2010;50:294-9.

[33] Krishna PD, Shankar PM, Newhouse VL. Subharmonic generation from ultrasonic contrast agents. Phys Med Biol 1999;44:681-94.

[34] Sijl J, Dollet B, Overvelde M, Garbin V, Rozendal T, de Jong N, et al. Subharmonic behavior of phospholipid-coated ultrasound contrast agent microbubbles. J Acoust Soc Am 2010;128:3239-52.

[35] Shekhar H, Rychak JJ, Doyley MM. Modifying the size distribution of microbubble contrast agents for high-frequency subharmonic imaging. Med Phys 2013;40:082903. 